\title{
THz Hot-Electron Bolometer Mixers
}

\author{
Sergey Cherednichenko, Stella Bevilacqua, and Evgenii Novoselov \\ Chalmers University of Technology, Göteborg, Fysikgränd 3, 41296 Sweden
}

\begin{abstract}
We overview the technology of superconducting Hot-Electron Bolometer mixers in the field of terahertz heterodyne detection. HEB mixer performance is analyzed vs competing technologies and application fields, with emphasize on radio astronomy. We discuss materials which have been reported in the past to be used for such devices with both phonon-substrate and electron-diffusion cooling mechanisms. It caused a splash of interesting theories with attempts of describing both de and RF characteristics of HEB mixers. Development of HEB mixers made possible to extend high spectral resolution radio astronomy beyond $1 \mathrm{THz}$.
\end{abstract}

\section{INTRODUCTION}

$\mathrm{S}$ UPERCONDUCTING Hot-Electron Bolometer (HEB) mixers are currently a crucial component in $\mathrm{THz}$ electronics [1]. This is essentially important for $\mathrm{THz}$ radio astronomy, where the presence of HEB mixers has opened a whole new range of possibilities for observations [2].

The idea of using thermal effects in thin films to extract the beating frequency of two electromagnetic waves has become serious for mixing application after discovery that the electron temperature relaxation time, $\tau$, in thin $\mathrm{Nb}$ films can be as short as just a few nanoseconds [3]. It means that the beating frequency (the intermediate frequency in the mixer terminology) can be as high as $1 /(2 \pi \tau) \sim 100 \mathrm{MHz}$. Later, even shorter electron temperature relaxation time $(10 \mathrm{ps})$ was discovered in thin $\mathrm{NbN}$ films [4]. Together with an idea of very fast electron diffusion in $\mathrm{Nb}$ nano-bridges [5] it gave a hint that $\mathrm{THz}$ mixers with a gain bandwidth of a few $\mathrm{GHz}$ can potentially be made using superconducting bolometers.

The word bolometer was justified by the fact that it is a direct transformation of the $\mathrm{THz}$ wave energy into a temperature rise of a superconducting micro (nano) bridge which takes place here, and that temperature rise is sensed via sharp resistance vs temperature dependence at the critical temperature of the superconductor. Though the HEB mixer operation turned out to be much more complex than it was believed that time, this simple description is still valid in general.

\section{MIXER SENSITIVITY}

The major figure of merit for low noise mixers is the mixer noise temperature, defined as a temperature of a blackbody source at the input of the mixer causing a factor of two rise of the mixer output noise as compared to the absolute zero blackbody source. Zero temperature quantum oscillations make this definition a bit more complex [6], and it becomes more important for frequencies well above $1 \mathrm{THz}$ and input signal levels below $100 \mathrm{~K}$. The mixer conversion loss (the loss of power during the signal conversion from the RF (Terahertz) to the IF $(\mathrm{GHz})$ ) is usually about $10 \mathrm{~dB}$. This fact imposes restrictions on the noise IF readout chain (determined by the input noise of the first Low Noise Amplifier (LNA). The state of the art LNAs covering a range of $2-4 \mathrm{GHz}$ or $4-8 \mathrm{GHz}$ have a noise temperature in a range of $2-5 \mathrm{~K}$. Most frequently the $\mathrm{THz}$ mixer performance is given in the units of a receiver noise temperature, which includes the noise from the IF LNAs, and sometimes the noise from the input optical loss. The later one is common for cryogenic mixers, like HEBs and SIS. A number of solutions exists for minimization and /or calibration for the input optics loss.

The most common materials used for THz HEB mixers are $\mathrm{NbN}$ and $\mathrm{NbTiN}$ thin films. With a $T_{c}$ on the order of $10 \mathrm{~K}$ and film thickness of $5 \mathrm{~nm}$ a gain bandwidth of $1-4 \mathrm{GHz}$ is readily available. The noise temperature, as the major figure of merit, is about $500-1500 \mathrm{~K}$ for signal frequencies up to $3-4 \mathrm{THz}[7,8$ , 9,10$]$. A summary of the receiver noise temperatures for THz receivers based on HEB, SIS, and Schottky diode mixers is given in Fig.1. The results for the Schottky diode mixers are for a room temperature operation $(290 \mathrm{~K})$, whereas for both the HEB and SIS the operation temperature is $4.2 \mathrm{~K}(\mathrm{LHe})$ or $2 \mathrm{~K}$ (pumping away the LHe vapors).

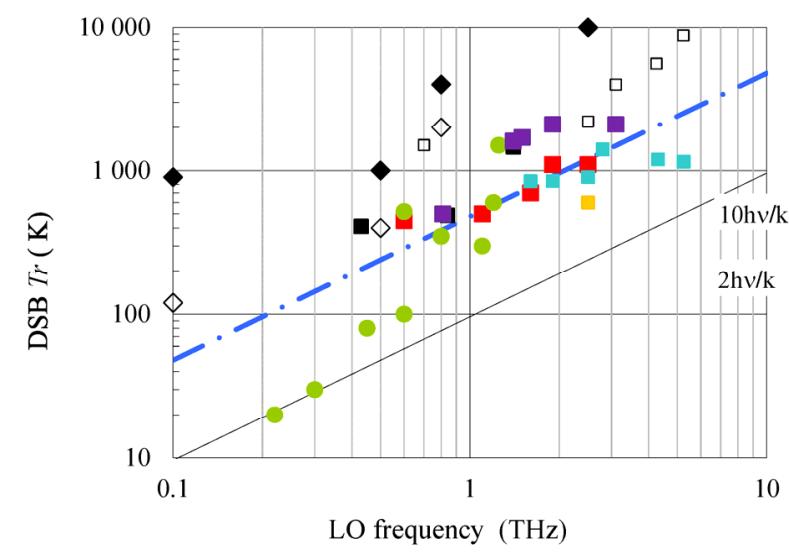

Fig. 1. Summary of the receiver Double Sideband noise temperature versus the Local Oscillator frequency based on HEB (squares), SIS (circles), and Schottky diode (diamonds) mixers. HEBs: different colors show results from different research groups. Schottky diodes: filled diamonds -room temperature, open diamonds- cryogenic. The solid and the dash-dot lines indicate the $\times 4$ and the $\times 20$ of the quantim noise limit $h v / 2 k B=24 \mathrm{~K} / \mathrm{THz}$. Refs are given for HEBs only.

In the literature, the HEB noise temperature is often referred to an IF in the range of $1-2 \mathrm{GHz}$, which is the IF range where the noise temperature is the lowest. It rises for higher IFs which is due to a limited HEB mixer gain bandwidth. Examples of the receiver noise temperature spectra for NbN HEB mixers across a $2-4 \mathrm{GHz}$ IF band are given in Fig.2, for a $1.6 \mathrm{THz}$ and a $1.9 \mathrm{THz} \mathrm{LO}$ frequencies.

Two RF schemes are utilized in order to match a sub micrometer size HEB mixer to a $\mathrm{THz}$ wave with a wavelength 


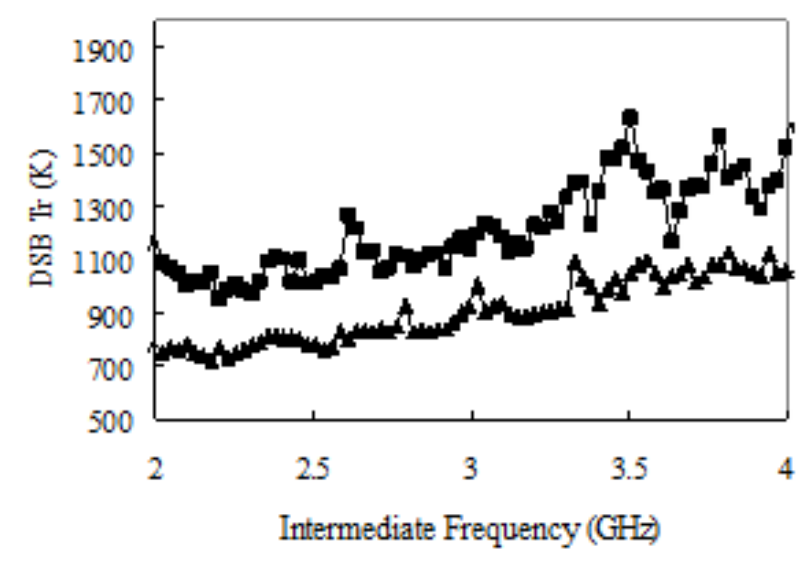

Fig. 2. NbN HEB Receiver noise temperature across the 2-4GHz IF band at $1.6 \mathrm{THz}$ (triangles) and $1.9 \mathrm{THz}$ (squares) LO frequencies. []

on an order of $100 \mu \mathrm{m}(3 \mathrm{THz})$ : a horn antenna with a mixer in a fundamental rectangular waveguide (waveguide mixers); or with a planar antenna on a silicon lens (quasioptical mixers) (see Fig.3). Both methods have their own advantages and disadvantages. For frequencies above $2 \mathrm{THz}$ it is antenna integrated HEB mixers so far have been reported. At the frequencies where both the waveguide and the quasioptical mixers are used, the mixers sensitivity is approximately the same.

The conversion loss of the HEB mixers increases as $L\left(f_{I F}\right)=L(0) \times\left(1+\left(2 \pi \times f_{I F} \times \tau\right)^{2}\right)$, where $f_{I F}$ is the IF, $L(0)$ is the conversion loss at the zero IF, and $\tau$ is the electron temperature relaxation time, as discussed above. At an IF equal to $f_{0}=1 /(2 \pi \times \tau)$, the $L\left(f_{I F}\right)$ equals to $L(0) \times 0.5$. The $f_{0}$ is called the mixer gain bandwidth (GBW). Due to the increase of the mixer conversion loss, the mixer noise temperature increases too, as shown in Fig.2. At higher IF the noise temperature rises even faster (Fig.4, blue crosses)) and no mixer performance has been reported for IF above $8 \mathrm{GHz}$.

For a practical application, other mixer/receiver have to to be also considered, such as the required Local Oscillator (LO) power, the stability, IF matching (IF ripples). Many publica-

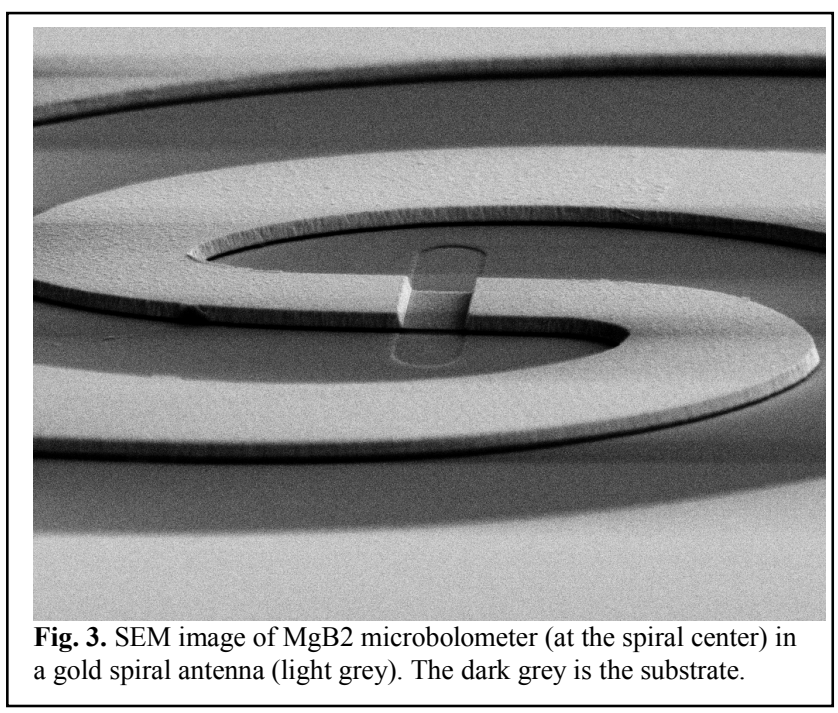

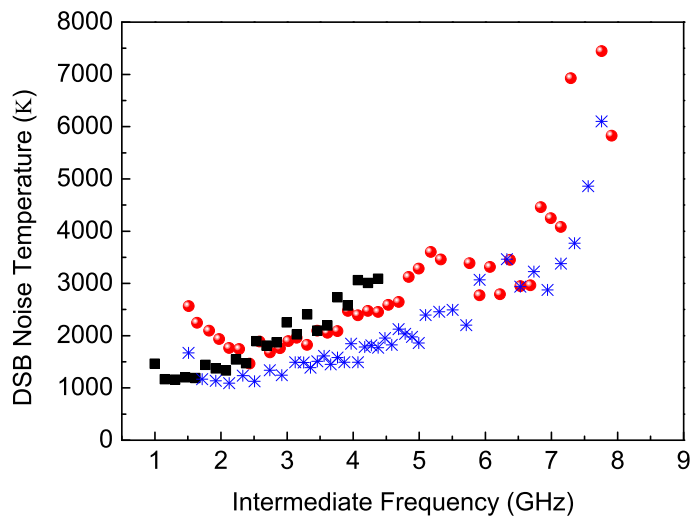

Fig. 4. The IF spectrum of the HEB mixer noise temperature for $\mathrm{NbN}$ (blue crosses), $10 \mathrm{~nm} \mathrm{MgB} 2$ ( $\mathrm{Tc}=8.5 \mathrm{~K}$, black squares), and $10 \mathrm{~nm}$ $\mathrm{MgB}_{2}(\mathrm{Tc}=15 \mathrm{~K}$, red squares $)$.

tions have been to those issues [11]. The LO power required to achieve the reported mixer noise temperature was one of the limiting factors for radio astronomical applications. The output power of the multiplier based LO sources (frequency upconversion from the microwave range to $\mathrm{THz}$ ) reduces to just a few microwatts for frequencies above $1 \mathrm{THz}$. In order to minimize the required LO power, HEB mixes were reduced in size to less than a $1 \mu \mathrm{m}^{2}$.

\section{SEARCH FOR NEW MATERIALS}

Despite of very good performance of NbN HEB mixers, a number of other materials have also been considered: $\mathrm{Nb}, \mathrm{Al}$, NbTiN, $\mathrm{MgB}_{2}$, MoRe. Both $\mathrm{Nb}$ and $\mathrm{Al}$ HEBs have demonstrated a GBW superior to NbN HEBs, however their noise temperature was much worse, making them impractical. NbTiN thin films with a $T c$ higher than for $\mathrm{NbN}$ were available from a large experience with SIS mixers. NbTiN HEB mixers [12] have been reported to have performance comparable to the $\mathrm{NbN}$ HEBs at lower IF (1-2GHz). However, the gain bandwidth (and, hence the noise bandwidth) for NbTiN HEB mixers were found to be much less compared to NbN HEBs (see Fig.5).

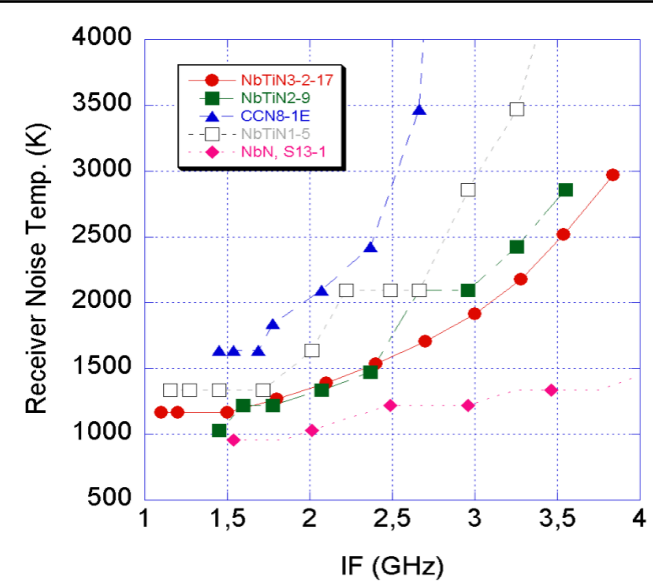

Fig. 5. A comparison of the DSB noise temperature of an NbN HEB (red diamonds) versus NbTiN HEBs receiver. 
More recently, HEB mixers made of $\mathrm{MgB}_{2}$ films $10-15 \mathrm{~nm}$ thick and with a $T_{c}$ of up to $15 \mathrm{~K}$ have demonstrated promising performance [13]. The minimum noise temperature was $600 \mathrm{~K}$ at $0.6 \mathrm{THz} \mathrm{LO}$ for devices made of $10 \mathrm{~nm} \mathrm{MgB}_{2}$ films with a Tc of $8.5 \mathrm{~K}$ [14]. A GBW of $3.4 \mathrm{GHz}$ was achieved for a $10 \mathrm{~nm}$ films with a Tc of $15 \mathrm{~K}$. An IF noise spectra for an $\mathrm{MgB}_{2}$ mixer versus $\mathrm{NbN}$ mixers is given in Fig.4.

The Tc of $\mathrm{MgB}_{2}$ films is higher compared to all other materials reported before for HEBs. Even for a relatively high Tc $\mathrm{NbN}$ HEBs the mixer noise temperature starts to rise almost immediately for temperatures above $4 \mathrm{~K}$. However, for an $\mathrm{MgB}_{2} \mathrm{HEB}$ mixer a constant noise temperature was observed way up to $10 \mathrm{~K}$ [15]. This fact opens prospects for HEB mixer operation at temperatures higher than LHe. This becomes even more interesting after $10 \mathrm{~nm} \mathrm{MgB}_{2}$ films with a Tc above $30 \mathrm{~K}$ have been reported.

\section{CONCLUSIONS}

In this paper we described some the most crucial aspects of $\mathrm{HEB}$ mixer technology. $\mathrm{NbN}$ and $\mathrm{NbTiN}$ thin films remain the only materials used for practical application in radio astronomy. Their noise temperature is just a few times the quantum limit. The useful IF range of the $\mathrm{NbN}$ and $\mathrm{NbTiN}$ HEB mixers is $1-5 \mathrm{GHz}$ and $1-3 \mathrm{GHz}$ correspondingly. It is also clear that in order to extend the IF range of HEB mixers to $10 \mathrm{GHz}$ new materials as needed. In this direction, thin $\mathrm{MgB}_{2}$ films with a high Tc $(>15 \mathrm{~K})$ could be of high interest.

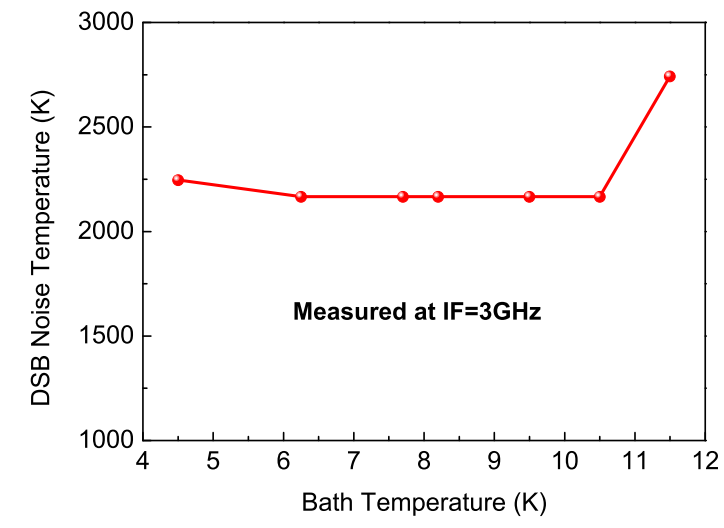

Fig. 6. DSB noise temperature of an $\mathrm{MgB}_{2} \mathrm{HEB}$ receiver vs operation temperature. LO frequency is $0.6 \mathrm{THz}$, the IF is $3 \mathrm{GHz}$.

\section{REFERENCES}

1 J. Zmuidzinas and P. L. Richards, "Superconducting detectors and mixers for millimeter and submillimeter astrophysics," Proc. IEEE, vol. 92, no. 10, pp. 1597-1616, Oct. 2004.

2 T. de Graauw et al., "The Herschel-heterodyne instrument for the farinfrared (HIFI)," Astron. \& Astrophys., vol. 518, p. L6, 2010.

3 E. M. Gershenzon, G. N. Gol'tsman, I. G. Gogidze, A. I. Elant'ev, B. S. Karasik, and A. D. Semenov, "Millimeter and submillimeter range mixer based on electronic heating of superconducting films in the resistive state," Sov. Phys. Supercond., vol. 3, no. 10, pp. 1582-1597, Oct. 1990
4 Y. Gousev, G. Goltsman, A. Semenov, and E. Gershenzon, J. Appl. Phys. 75, 3695 _1994.

5 D. E. Prober, "Superconducting terahertz mixer using a transition-edge microbolometer"'Appl. Phys. Lett. 62, 2119 (1993).

6 E.L.Kollberg, and K.S. Yngvesson, " Quantum-Noise Theory for Terahertz Hot Electron Bolometer Mixers”, IEEE Trans. Microw. Theory Techn., vol. 54, no. 5, pp. 2077-2089, May. 2006

7 S.Cherednichenko, V.Drakinskiy, T. Berg, P. Khosropanah, and E. Kollberg "Hot-electron bolometer terahertz mixers for the Herschel Space

Observatory", Rev. Sci. Instrum. 79, 034501 (2008).

8 I. Tretyakov, S. Ryabchun, M. Finkel, A. Maslennikova, N. Kaurova, A. Lobastova, B. Voronov, and G. Gol'tsman, "Low noise and wide bandwidth of NbN hot-electron bolometer mixers," Appl. Phys. Lett., vol. 98, no. 3, p. 033507-3, Jan. 2011

9 W. Zhang, P. Khosropanah, J. R. Gao, E. L. Kollberg, K. S. Yngvesson, T. Bansal, R. Barends, and T. M. Klapwijk, " Quantum noise in a terahertz hot electron bolometer mixer", Appl. Phys.Lett., 1111132010

10 A. D. Semenov, H. W. Hubers, H. Richter, M. Birk, M. Krocka, U. Mair, Y. B. Vachtomin, M. I. Finkel, S. V. Antipov, B. M. Voronov, K. V. Smimov, N. S. Kaurova, V. N. Drakinski, and G. N. Gol'tsman, "Superconducting HotElectron Bolometer Mixer for Terahertz Heterodyne Receivers", IEEE Trans. Appl.Supercond. Vol.13, 2, 2003, pp.168-171

11 I J. J. A. Baselmans, M. Hajenius, J. R. Gao, A. Baryshev, J. Kooi, T. M. Klapwijk, B. Voronov, P. de Korte, and G. Gol'tsman, "NbN hot electron bolometer mixers: sensitivity, LO power, direct detection and stability," IEEE Trans. Appl. Supercond., vol. 15, no. 2, pp. 484-489, Jun. 2005

12 L. Jiang, S. Shiba, K. Shimbo, N. Sakai, T. Yamakura, M. Sugimura, P. G. Ananthasubramanian, H. Maezawa, Y. Irimajiri, and S. Yamamoto,

"Development of THz waveguide. NbTiN HEB mixers," IEEE Tran. Appl. Supercond., vol. 19, no. 3, pt. 1, pp. 301-304, Jun. 2009

13 S.Cherednichenko, V.Drakinskiy, K.Ueda, T.Naito "Terahertz mixing in $\mathrm{MgB}_{2}$ microbolometers", Applied Physics Letters, 90 ( 2 ) pp, (2007)

14 S. Bevilacqua, S. Cherednichenko, V. Drakinskiy, J. Stake, H. Shibata, and Y. Tokura, "Low noise $\mathrm{MgB}_{2}$ terahertz hot-electron bolometer mixers", Appl.Phys.Lett., 100, 033504 (2012)

15 S. Bevilacqua, S. Cherednichenko, V. Drakinskiy, H. Shibata, Y. Tokura . "Study of IF bandwidth of $\mathrm{MgB}_{2}$ phonon-cooled hot-electron bolometer mixers". IEEE Trans. on Terahertz Sci. Technol.. v.3, n.4,pp. 409-415, Jul. 2013 